\title{
Large Area Microfluidic Mixer \\ Integrated with Linear Fluidic Transporters and Reservoirs
}

\author{
Hongyu Yu and Eun Sok Kim \\ Department of Electrical Engineering-Electrophysics \\ University of Southern California \\ Los Angeles, CA 90089-0271
}

\begin{abstract}
This paper describes a large area (over $100 \mathrm{~mm}^{2}$ ) micromixer integrated with long-channel fluidic transporters and reservoirs on a same substrate. Both the micromixer and the linear fluid transporter are built on a PZT sheet with its electrodes patterned to produce self-focused acoustic beams for powerful inplane liquid motion (through acoustic streaming effect). Selffocusing Acoustic Transducers (SFAT) are sectored and connected in various patterns for a variety of mixing and transport effects, and are set under a silicon, glass or plastic wafer that contains a large area, shallow, mixing chamber. Mixing in a $200 \mu \mathrm{m}$ deep, $100 \mathrm{~mm}^{2}$ chamber can be done within 2 seconds.
\end{abstract}

\section{INTRODUCTION}

Lab-on-chip (LOC) with microfluidic processing capabilities has great potential for biomedical applications. Especially, microfluidic systems that can process liquids of tens of $\mu \mathrm{l}$ are greatly needed for many biomedical processing systems. Microfluidic mixing has conventionally been done using heat, electrolysis and electromagnetic force. These methods, however, present some difficulties in any of the following requirements: compatibility with biomedical applications, easiness of integration with LOC system, mixing capability of relatively large volume liquid. It has been reported that focused acoustic waves are effective in generating fluidic motion [1], and a sectored Selffocusing Acoustic-wave Transducer can produce strong in-plane liquid motion for micromixing [2]. Thus, we have used several arrays of sectored SFATs to transport (over tens of mm length) and mix (over hundreds of $\mathrm{mm}^{2}$ ) fluids noninvasively. This paper describes the theory, design and experimental results of the fluid mixer integrated with fluidic transporters and reservoirs.

\section{THEORY}

SFAT with its electrodes on both faces of a piezoelectric film patterned as shown in Fig.1 focuses acoustic waves through constructive wave interference [3]. When RF power is applied between the electrodes (sandwiching the piezoelectric film) with its frequency corresponding to the thickness mode resonance of the piezoelectric film, strong acoustic waves are generated over the areas covered by the electrodes. With a proper design of the electrode patterns (e.g., a set of annular rings acting as half-waveband sources), we can achieve wave-focusing effect without any acoustic lens due to the acoustic waves interfering with each other as they propagate in the fluid adjacent to the transducer.

With a set of complete annular rings, the acoustic field is radially symmetrical in the plane of the rings. But when the rings are broken into segments of various angles, the acoustic field becomes nonsymmetrical in the in-plane. The basic cell that we employ for the SFAT fluid driver is a $90^{\circ}$ sectored SFAT built on a PZT substrate shown in Fig. 2.

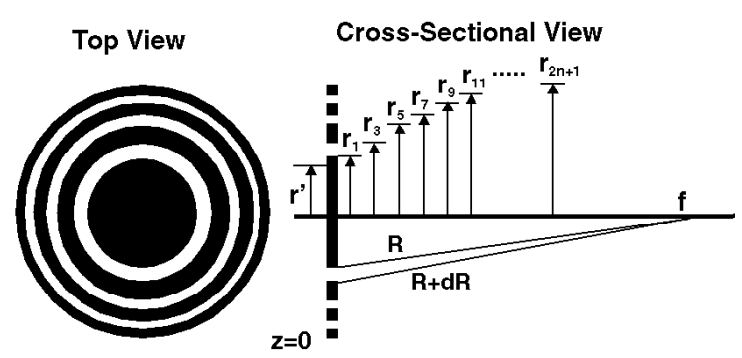

Annular Rings: Half-Wave-Band Sources

$$
r_{n}=\sqrt{\frac{q \lambda}{2}\left(f+\frac{q \lambda}{8}\right)} \quad(q=n+1+2 \cdot \text { offset })
$$

Fig. 1 The electrode pattern of annular rings on both faces of a piezoelectric plate for self-focusing of acoustic waves.

A high-intensity acoustic wave propagating in a medium is absorbed and scattered by the medium. The wave attenuation with a high intensity wave is nonlinear, and causes the medium itself to move. This nonlinear acoustic effect is called acoustic streaming.

In a sectored SFAT, the electrode patterns for the top and bottom electrodes are designed to produce large acoustic pressure gradient (especially in the in-plane direction) near the focal points. As the intensified acoustic waves travel in the liquid with accompanying acoustic loss, the acoustic streaming happens, and there exists steady body force in the liquid to drive fluid flow [4].

$$
\vec{F}_{b}=\rho_{0}\left\langle\vec{v}_{1} \nabla \cdot \vec{v}_{1}+\left(\vec{v}_{1} \cdot \nabla\right) \vec{v}_{1}\right\rangle
$$

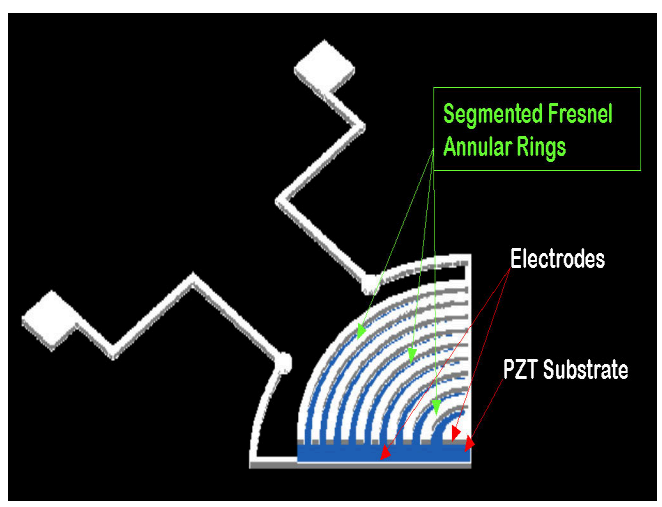

Fig. 2 A $90^{\circ}$ sectored SFAT cell 


\section{FABRICATION}

Figure 3 shows the fabrication process for the sectoredSFAT fluid driver. We use a $192 \mu \mathrm{m}$ thick PZT sheet as the transducer substrate. With the front-to-backside alignment marks, $2.54 \mu \mathrm{m}$ thick silver electrodes on both sides of the PZT sheet are patterned such that the top and bottom FASA electrodes overlap each other. The silver is etched with $3 \mathrm{M} \mathrm{HNO}_{3}$ in 35 seconds. The fluid chambers are micromachined in silicon substrate using HNA isotropic etching or $\mathrm{KOH}$ anisotropic etching. Two kinds of mixing chambers have been fabricated: one in a $400 \mu \mathrm{m}$ thick silicon wafer, the other over a $170 \mu \mathrm{m}$ thick glass or $100 \mu \mathrm{m}$ thick plastic wafer with an etched-through silicon on its top (shown in Fig 4). The mixing chambers, built on a silicon wafer, are fabricated together with linear channels and rectangular reservoirs on a same substrate to produce an integrated microfluidic subsystem, which is then bonded (with about $10 \mu \mathrm{m}$ thick epoxy) to the PZT transducer (Fig.3).

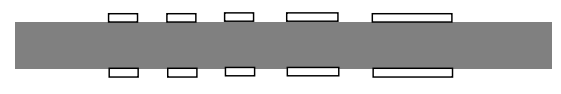

1. Pattern electrodes on a PZT sheet

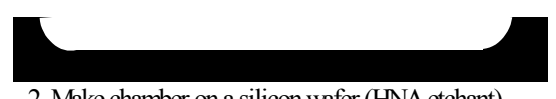

2. Make chamber on a silicon wafer (HNA etchant)

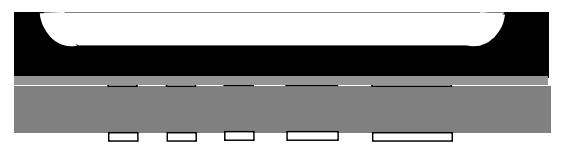

3. Attach the PZT sheet to the Si wafer with epoxy.
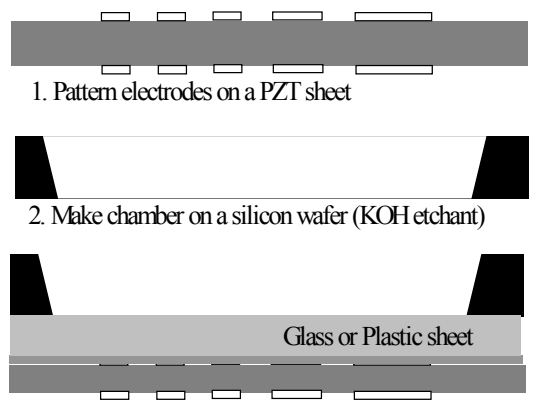

3. Attach the PZT sheet to the Glass/Plastic wafer and Silicon chamber with epoxy.

Fig. 3 Brief fabrication steps for two types of mixing chambers in Si wafer (top three) and Glass or plastic wafer (bottom three).

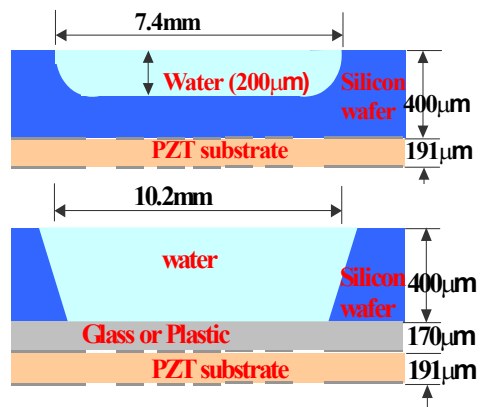

Fig. 4 Cross sectional views of the two types of mixing chambers.

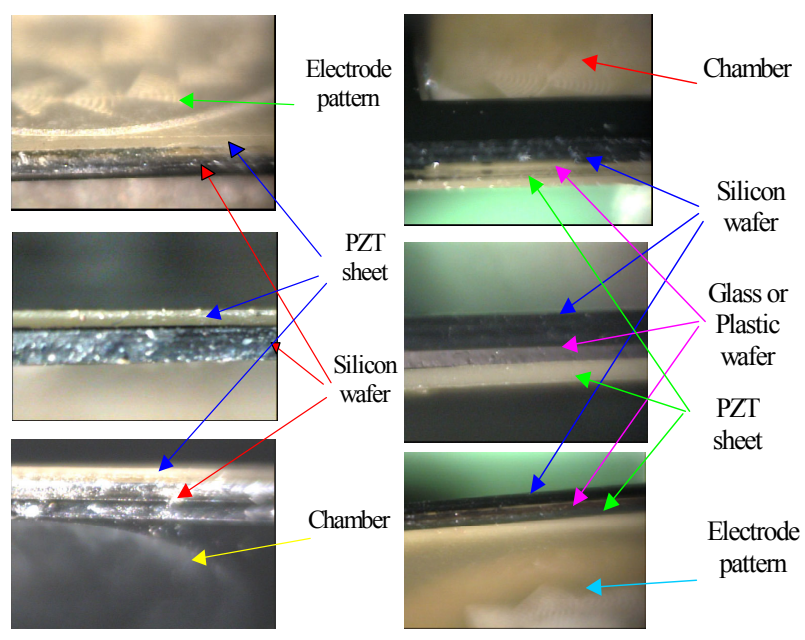

Fig. 5 Cross-sectional photos of the completed mixers

\section{EXPERIMENTAL METHOD}

The fabricated devices are tested in an apparatus shown in Fig. 6, where a RF square wave (with the frequency around 12 $\mathrm{MHz}$ corresponding to the thickness of PZT sheet) is modulated with a sequence of pulses from a function generator before being amplified by an RF amplifier and applied to the device. The peakto-peak voltage of the RF amplifier output is typically about $40 \mathrm{~V}$, and the electrical field across the PZT substrate is about $10^{5} \mathrm{~V} / \mathrm{m}$. When we apply a continuous square wave, the series resistance of the electrodes produce significant amount of heat. Thus, we use a pulsed square wave with 30 to $120 \mathrm{~Hz}$ pulse repetition frequency and on time of 40 to $100 \mu \mathrm{s}$ (corresponding to a duty cycle of $0.12 \%$ to $1.2 \%$ ). With this pulsed wave, heating is negligible.

The fluidic subsystem includes a mixer, two linear transporters and rectangular reservoirs. In the rectangular reservoirs, we first place red ink and DI water along with microspheres of $10 \mu \mathrm{m}$ in diameter to facilitate the observation of the liquid flow and mixing trace. Those liquids are transported from the reservoirs to the circular mixing chamber through the connecting fluidic channels by the microfluidic driver [5], and then are mixed together in the mixing chamber by the micromixer.

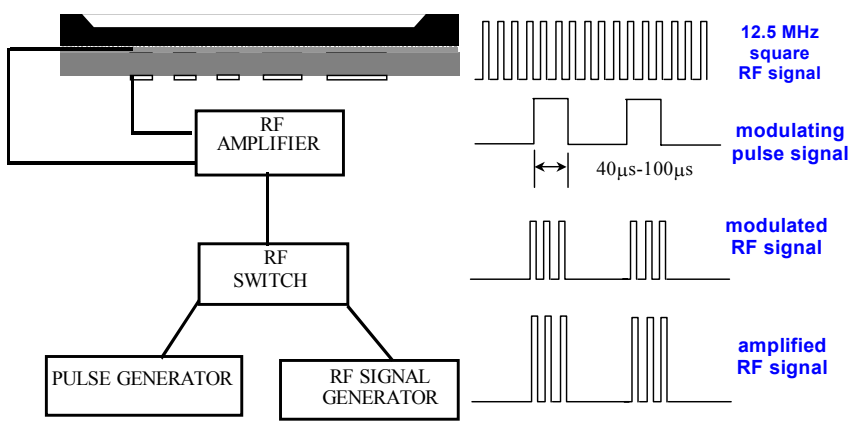

Fig. 6 Schematic of the test set-up.

\section{EXPERIMENTAL RESULTS}

The top-view photo of the fabricated microfluidic subsystem (with two rectangular reservoirs of $9.4 \times 3.4 \mathrm{~mm}^{2}$ each, $1 \mathrm{~mm}$ wide and $8.4 \mathrm{~mm}$ long channels, and a circular mixing 
chamber of $7.4 \mathrm{~mm}$ in diameter) is shown in Fig. 7. The micromixer can mix liquids (about 0.2 to $0.4 \mathrm{~mm}$ tall) over $100 \mathrm{~mm}^{2}$ area within 2 seconds. To demonstrate the mixing capability, two kinds of liquids (red ink and DI water) with microspheres are stored in the two rectangular reservoirs (on the right-hand side of Fig. 7), driven along the two narrow and long channels (on the middle of Fig. 7) by linear arrays of the sector SFATs, and mixed in the circular chamber (on the left-hand side of Fig. 7) by the micromixer.

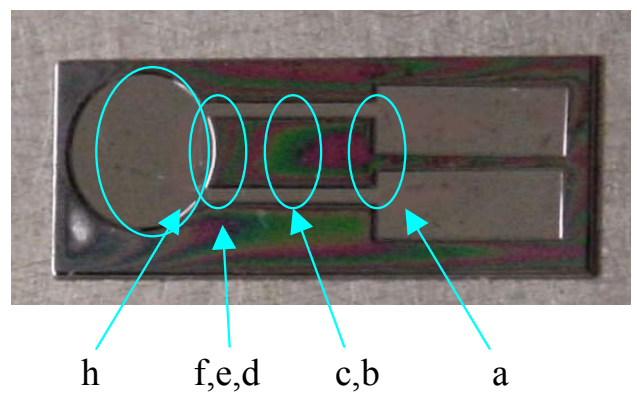

Fig. 7 Top view photo of the fabricated microfluidic subsystem made in a silicon wafer with PZT sheet.

The fluids in the subsystem are driven by the sector SFATs, and their flows are captured at the four regions indicated with elliptical circles in Fig. 7 at different points of time (a-h) as follows:

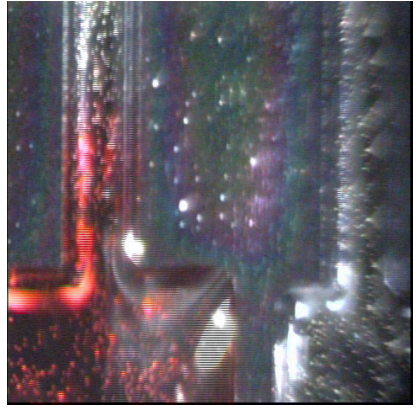

a

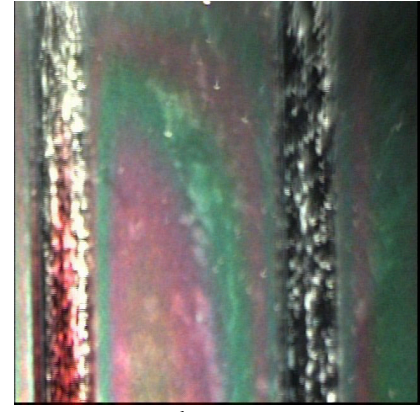

b

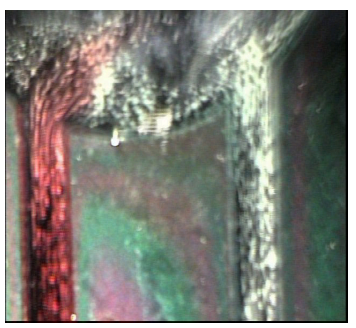

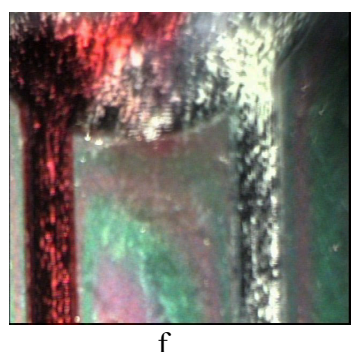

(e) $1.7 \mathrm{sec}$ after the turn on of the pulses, the liquids arrive at the mixing chamber;

(f) start of the mixing;

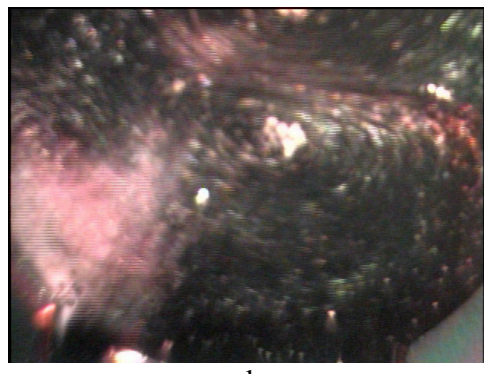

$\mathrm{h}$

(h) $3.2 \mathrm{sec}$ after the power on (it shows the effective mixing of the two liquids).

The mixing speed can easily be controlled by adjusting the pulse duty cycle or the peak voltage level. And different electrode patterns on the SFAT can produce different fluid mixing traces as shown in Fig. 8: in (a) there are 3 "eyes" producing almost random mixing, while in (b) there is only one "eye" in fluid mixing flow which would be good for a directional mixing.
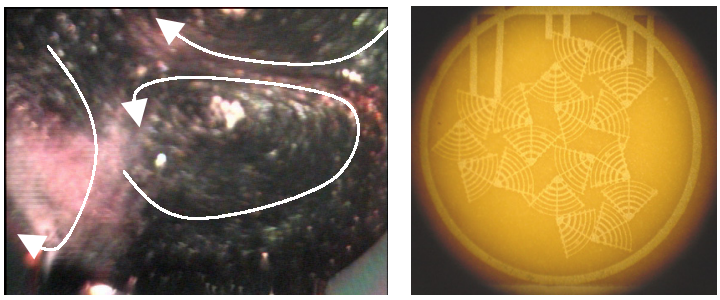

(a)
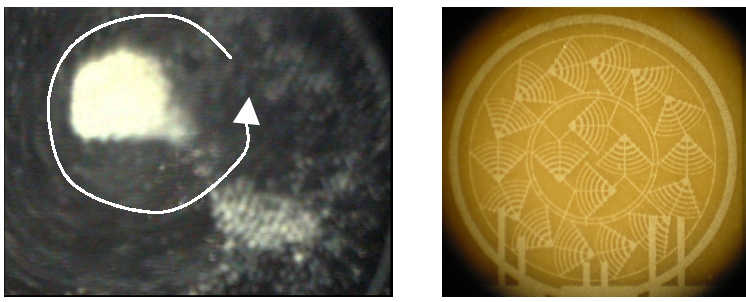

(b)

Fig. 8 Mixing patterns and the electrode patterns on the SFATs that produce the mixing traces.

In the experiment, we find the 'eye' point is not good for mixing liquids with particles, because the particles come and tend to stay there without moving around. To solve this problem, we adjust the RF frequency so that the liquid mixing trace may be changed. When the frequency is changed a little, such as $\pm 0.1 \mathrm{MHz}$, there is still good mixing effect in the chamber, although the mixing speed is slowed down a little, and at same 
time, the liquid mixing trace is changed. The reason for such trace change is that when the frequency is varied, the interference between the waves varies, resulting in a variation in the acoustic wave distribution in the liquid, which changes the body force distribution in the liquid,. When we change the liquid traces by changing the RF frequency, we can force all particles to mixing and avoid the nodal effect by the 'eye' (Fig.9).
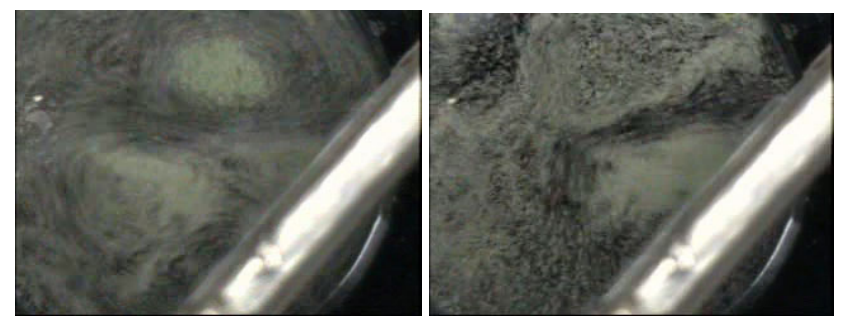

Fig. 9 Liquid trace changes when the $R F$ frequency is changed by a small amount.

When the PZT SFAT is glued to a silicon wafer that contains microfluidic channels and chambers, the S11 parameter of the SFAT (without any liquid) is as shown in Fig. 10. There are two peaks in the S11 (due to the micromachined silicon attached to the PZT sheet), which broaden the resonance characteristic. From the $\mathrm{S} 11$, we see that there is still at least $30 \%$ of the peak power at the frequency $\pm 0.1 \mathrm{MHz}$ off from the center frequency. With liquid in the mixing chamber, the drop in the available power (from the peak power as the frequency is varied) would be less due to the mass loading effect by the liquid. Thus, we would still have significant amount of acoustic power available for mixing, even when we vary the RF frequency to change the liquid traces.

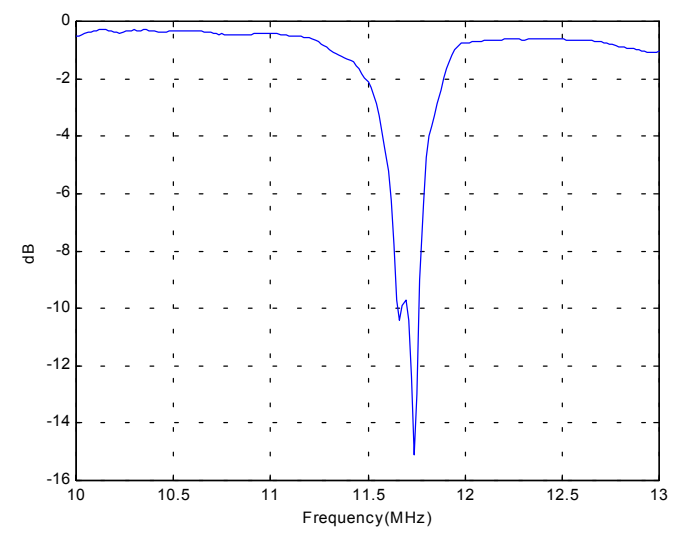

Fig. 10 S11 parameter of the sector SFAT attached to a silicon wafer with microfluidic channels and chambers.

In addition to silicon wafer, glass and plastic wafers also have been used as the chamber/channel/reservoir substrate. We get almost same mixing effect on these wafers, although the mixing speeds are slowed down, especially on the plastic wafer, due to more acoustic absorption in the plastic or glass wafer than in a silicon wafer. Figure 11 shows the time sequence of the mixing effect on a plastic wafer.

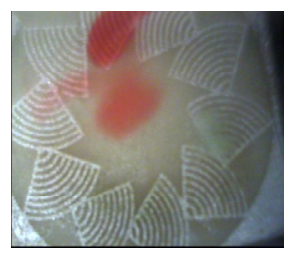

a

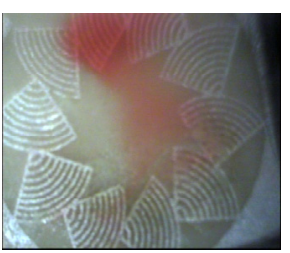

b

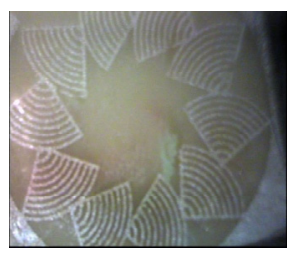

$\mathrm{c}$
Fig 11 Time procession of the mixing: (a) Right after red ink is dropped in the water, (b) $2.4 \mathrm{sec}$ later, (c) $8.6 \mathrm{sec}$ later.

\section{SUMMARY}

In this paper, we describe a large area (over $100 \mathrm{~mm}^{2}$ ) micromixer integrated with long-channel fluidic transporters and large reservoirs on a same substrate. The three major microfluidic components are integrated in a micromachined silicon substrate with sector SFATs built on a PZT sheet. The micromixers and linear fluid transporters built on the PZT SFATs produce strong inplane fluid motion without heat. And we demonstrate a powerful mixing effect over a large surface area. In addition, we demonstrate the integrated microfluidic subsytem over a glass or plastic wafer with silicon sidewalls. The microfluidic subsystem described in this paper integrates three major fluidic components (i.e., micromixer, liquid transporter, and reservoir), and is ready for a stand-alone microfluidic system, once one-way valves are integrated.

\section{ACKNOWLEDGMENT}

This material is based upon work supported by Defense Advanced Research Projects Agency under contract \#N66001-00C-8094.

Travel support has been generously provided by the Transducers Research Foundation and by the DARPA MEMS and DARPA BioFlips programs.

\section{REFERENCES}

[1]. X. Zhu and E.S. Kim, "Microfluidic Motion Generation with Acoustic Waves," Sensors and Actuators: A. Physical, vol. 66/1-3, pp. 355-360, April 1998.

[2] V. Vivek, Y. Zeng and E.S. Kim, "Novel Acoustic-Wave Micromixer," IEEE International Micro Electro Mechanical Systems Conference, Miyazaki, Japan, January 23-27, 2000, pp. 668-673.

[3] D. Huang and E.S. Kim, "Micromachined Acoustic-Wave Liquid Ejector," IEEE/ASME Journal of Microelectromechanical Systems, vol. 10, pp. 442-449, September 2001

[4]. T. Uchida, T. Suzuki and S. Shiokawa, "Investigation of Acoustic Streaming Excited by Surface Acoustic Waves," 1995 IEEE Ultrasonics symposium pp. 1081-1084.

[5] H. Yu and E.S. Kim, "Noninvasive Acoustic-Wave Microfluidic Driver," IEEE International Micro Electro Mechanical Systems Conference, Las Vegas, Nevada, January 2024, 2002, pp.125-128. 